\title{
The Role of Multimodality Imaging Techniques on Differential Diagnosis of Precancerous Nodules and Hepatocellular Carcinoma
}

\author{
Hui-Jie Jiang ${ }^{1}$, Xiao-Feng $\mathrm{Li}^{2}$ \\ ${ }^{1}$ Department of Radiology, The Second Affiliated Hospital of Harbin Medical University, Harbin, China \\ ${ }^{2}$ Department of Diagnostic Radiology, University of Louisville School of Medicine, Louisville, USA \\ Email: jhjemail@163.com, xiao-feng.li@louisville.edu
}

Received September 5, 2012; revised October 12, 2012; accepted October 23, 2012

\begin{abstract}
In recent years, the incidence of hepatocellular carcinoma (HCC) has been increasing worldwide, and its high mortality seriously threatens public health. Early detection and treatment are crucial to improving the survival rate. Imaging examination widely used for the diagnosis of HCC and provides a non-invasive means of tumor visualization. The rapid development of medical imaging technology is expected to improve early-stage diagnosis rates for HCC. This article summarizes the methods for the differential diagnosis of pre-malignant dysplastic nodule (DN) and small hepatocellular carcinoma during the carcinogenesis of cirrhosis and reviews their application. In addition, a discussion on some recently patented medical imaging development was also presented.
\end{abstract}

Keywords: Precancerous Nodules; Small Hepatocellular Carcinoma; CT; MRI:Ultrasound

\section{Introduction}

Hepatocellular carcinoma (HCC) arises from cirrhosis through multi-levels of differentiation. This process produces regenerative nodules (RNs), low grade dysplastic nodules (LGDNs), and high-grade dysplastic nodules (HGDNs). The dysplastic nodule (DN) is a pre-malignant stage of primary HCC [1]. Small HCC refers to a cancerous nodule with a diameter $\leq 2 \mathrm{~cm}$, which can be divided into the early HCC and the progressive-stage small HCC [2]. We herein summarize methods for the differrential diagnosis of pre-malignant $\mathrm{DNs}$ and small $\mathrm{HCC}$ nodules and review their application.

\section{The Application of Ultrasound on Differential Diagnosis of DN and Small HCC Nodule}

\subsection{Traditional Ultrasound Examination Methods}

Conventional two-dimensional ultrasound has a low detection rate for $\mathrm{DN}$, only with a rate of $1.6 \%$ [3]. It also overlaps significantly with the echo from early HCC. Color Doppler flow imaging and pulsed Doppler techniques offer some value in the differentiation of $\mathrm{DN}$ and small HCC. Previous study thought that pulsatile blood flow in the nodules, pulsatile afferent arterial blood flow, and continuous efferent blood flow are typical color Doppler flow imaging manifestations of HCC [4]. Recent progresses in color power Doppler increase the detection rate of flow signals even in small HCCs $(76 \%-85 \%)[5,6]$.

For well-differentiated early $\mathrm{HCCs}$, it is difficult for color Doppler flow imaging to differentiate DN and early HCC, due to the presence of fewer arteries and low blood flow velocity. At the same time, Color Doppler flow imaging is unable to detect some small HCCs with less abundant blood supply. Color power angiography examination has a higher spatial resolution and signal/noise ratio and is more sensitive to low-speed blood flow in small blood vessels. It is affected to a lesser extent by the angle and the direction, and is not associated with an aliasing phenomenon. The sensitivity and specificity of color power angiography was considered to be similar to those of angiography. Three-dimensional color power angiography allows calculation of the percentage of tumor blood vessels in total volume; therefore, it may reflect the blood supply situation of the lesion more accurately than two-dimensional ultrasound. However, color power angiography and 3D-color power angiography cannot display the direction and speed of blood flow, and they provide no quantitative information. Moreover, they are vulnerable to the impact of organ activity and may generate artifacts. The aforementioned methods may fail to detect deep lesions, as well as lesions at the edge of 
the left lateral liver lobe and right sub-diaphragmatic lesions. Therefore, they have not been used widely for the early diagnosis of liver cancer in recent years.

\subsection{Contrast-Enhanced Ultrasound}

Previous studies have shown that contrast-enhanced ultrasound, computer tomography (CT), and magnetic resonance imaging (MRI) have the same sensitivity and specificity for screening and identifying small liver nodules [7-9]. It is generally believed that most of DNs are enhanced synchronically with the surrounding liver parenchyma [10]. In $20 \%$ of the lesions, the arterial phase is later than that of the surrounding liver parenchyma, and the remaining phases show equal enhancement, which may be related to complete or incomplete fibrous capsule formation in the DN. During degeneration, necrosis or significant inflammatory cell infiltration occurs in the DN, and ultrasound imaging may show no perfusion during the entire phase. The main contrast-enhanced ultrasound manifestations of small HCC are significant rapid enhancement during the arterial phase and excretion of the contrast agent during the portal venous and delayed phases, while the enhancement of the surrounding liver parenchyma is starting, and the echo intensity within the lesion is significantly lower than that of the surrounding liver parenchyma. It has been reported that such an enhancement pattern of "rapid filling-in and rapid washout" accounted for more than $80 \%$ of HCCs $[11,12]$. In HCC, the start time of contrast agent excretion is related to the degree of differentiation of nodular cells. In well-differentiated HCCs, the start time is later than in poorly differentiated HCCs. Therefore, well differentiated early HCCs often show an enhancement model of "rapid filling-in and slow washout" [13,14]. This may be because a well-differentiated early HCC often has a double blood supply from the portal vein and the hepatic artery [1]. During the arterial phase, the contrast agent is infused rapidly from the hepatic artery, and during the portal venous phase a large proportion of contrast agent is infused continuously through the portal vein, resulting in the histological basis for "rapid filling-in and slow washout".

Since 1990s, ultrasound imaging has evolved rapidly owing to new advances of ultrasound contrast agents, and its application in the early diagnosis of liver cancer has become increasingly widespread. Sonazoid is a new type of perfluorocarbon microbubble that remains in the liver parenchyma for a longer period of time [15]. The display can last from 60 to 120 minutes after injection. The examination can be completed when the mechanical index is low, which reduces the rate of microbubble destruction.

The stable Kupffer phase provided by Sonazoid (postvascular phase) overcomes the shortcomings of con- trast-enhanced ultrasound arising from the short duration of the arterial phase, which is not good for scanning the entire liver and detecting small lesions. At the same time, contrast-enhanced ultrasound offers good visibility without using sophisticated equipment. Its application has been generalized in clinical practice because of its reduced dependence on the operator's skill and equipment. Sonazoid is mainly engulfed by reticuloendothelial cells. Since there is an absence of Kupffer cells in malignant liver tumors, perfusion defects often occur in the Kupffer phase. Some studies proposed the "defect-reperfusion" approach for Sonazoid ultrasound imaging, in which the area of perfusion defect of the liver during the Kupffer phase was observed first, and then Sonazoid was re-injected [16]. At that time obvious arterial perfusion appears that is "defect-reperfusion positive", suggesting a high possibility of liver cancer. This method can be used for the detection of small HCC, and its sensitivity for the detection of small $\mathrm{HCC}$ is even higher than that of the multi-slice spiral CT.

The introduce of Sonazoid has expanded the use of ultrasound imaging [17] .which was previously used to differentiate between HCC and benign lesions, determine the degree of HCC differentiation, evaluate efficacy after transcatheter arterial chemoembolization, and guide fineneedle aspiration. Now it also has important roles in guiding RF ablation and detecting post-surgery relapsed lesions, the clinical staging of HCC, and the selection of treatment regimens.

\section{The Application of CT on Differential Diagnosis of DN and Small HCC Nodule}

\subsection{Plain CT and Three-Phase Enhancement CT}

It is generally believed that DNs appear as low-density or isodense nodules in a plain scan. Since the nodule is small and has characteristics similar to early-phase liver cancer, with its blood supply coming mainly from the portal vein, these two types of nodules often show synchronized enhancement with the liver parenchyma in enhanced scan and are difficult to be detected. $60 \%$ to $70 \%$ of DNs were isodense in three-phase dynamic contrast-enhanced CT [18], which means that only $35 \%$ of DN can be detected in contrast-enhanced CT. Although it has been reported occasionally that DNs show similar enhancement to liver cancer in the arterial phase, the incidence is rare [14]. For the DNs that have been detected, it is common that they appear as low-density nodules in the portal venous phase and equilibrium phase. Most early HCCs showed low or iso-density in the arterial phase or the delayed phase in contrast-enhanced CT [19]. And only $5 \%$ of early HCCs showed high density in the arterial phase. It can be seen that the CT characteristics of early HCCs and DNs overlap greatly, and it is 
very difficult to differentiate the two accurately. The main blood supply of small HCCs at the progressive stage is arterial, therefore, it has the typical enhancement feature of "rapid filling-in rapid washout". A small portion of hypovascular HCCs show no significant enhancement in the arterial phase, therefore, lack of enhancement in the arterial phase cannot be a criterion for ruling out malignant lesions.

\subsection{CT Hepatic Arteriography and CT Arterial Portography}

CT hepatic arteriography (CTHA) and CT arterial portography (CTAP) may reflect hemodynamic changes that occur during the carcinogenesis of cirrhosis and may help to differentiate benign and malignant lesions. Therefore, they are relatively sensitive methods for the early diagnosis of HCC. With increases in the malignancy of hepatic cirrhotic nodules and changes in the accompanying blood flow perfusion, CTHA and CTAP also show progressive changes. The CTHA and CTAP of hepatic cirrhotic nodules were classified into four types based on their CT features, which had corresponding histopathological types [20]. CTAP and CTHA provide an important guide to the early diagnosis of liver cancer and selection of clinical treatment regimen. They are especially valuable for lesions $\leq 3 \mathrm{~cm}$. However, their clinical application is limited due to trauma.

\subsection{CT Perfusion Imaging}

CT perfusion imaging is a functional CT imaging technique that can reveal morphological changes while reflecting in vivo hemodynamic changes and providing quantitative and semi-quantitative analysis. Common liver CT perfusion parameters are hepatic blood flow, hepatic blood volume, mean transit time of contrast agent, capillary permeability surface area product, hepatic arterial perfusion index, hepatic artery perfusion, and portal vein perfusion. Perfusion CT has a limitation when scanning small targets because the color mapping image may not enable visualization of a small lesion. To date, true perfusion imaging techniques have not been used for the surveillance of HCC or dysplastic nodules. Whole-liver CT imaging has the potential to provide both high-temporal-resolution and high-spatial-resolution imaging of the entire liver for the detection of small HCC [21]. With development of more advanced scanning, CT perfusion imaging will detect suspicious nodules based on liver cirrhosis to further differentiate benign and malignant nodules.

Compared with normal liver tissue or cirrhotic tissue, the main changes in $\mathrm{CT}$ perfusion parameters of $\mathrm{HCC}$ and surrounding infiltration areas are increases in hepatic artery perfusion, hepatic arterial index, hepatic blood flow, and hepatic blood volume, among which hepatic artery perfusion, hepatic arterial perfusion index provide the best diagnostic efficacy. It is reported that when the threshold of hepatic arterial index was set at 0.308 , the sensitivity of hepatic artery perfusion and hepatic arterial perfusion index in differentiating tumor and cirrhosis was $100 \%$, and specificity was $90 \%$.

Several studies showed that the HAP is significantly increased in $\mathrm{HCC}(0.94 \pm 0.26 \mathrm{ml} / \mathrm{min}$ per milliliter $)$. In progressive $\mathrm{HCC}$ and background liver tissue, $\mathrm{HBF}$ is higher in HCC tissue than in background liver tissue [22, 23]. Additionally, the growth characteristics of HCC can be evaluated by analyzing portal vein perfusion at the tumor edge, allowing an estimated prognosis for patients with liver cancer. Portal vein perfusion may also be beneficial for clinicians in selecting the appropriate range for liver resection.

\section{The Application of MRI on Differential Diagnosis of DN and Small HCC Nodule}

After the text edit has been completed, the paper is ready for the template. Duplicate the template file by using the Save As command, and use the naming convention prescribed by your journal for the name of your paper. In this newly created file, highlight all of the contents and import your prepared text file. You are now ready to style your paper.

\subsection{Unenhanced and Dynamic Contrast-Enhanced MRI}

Typical DN produces homogeneous high signals on T1weighted images (T1WI) and low signals on T2-weighted images (T2WI) in MRI. However, previous studies showed that only $33 \%$ of DNs showed high signals on T1WI and low signals on T2WI, and $67 \%$ of DNs were of the same intensity on T1WI and T2W [24]. Iron-containing DNs showed low signals on both T1WI and T2WI. DNs rarely show high signals on T2WI. When infarction occurs in DN, it may produce high signals on T2WI. Three-phase contrast enhanced MRI scans using gadolinium contrast agent exhibited no significantly different resolution from the surrounding liver parenchyma. In a small number of cases, enhancement in the arterial phase similar to that of HCC may appear, indicating that some DNs have increased blood supply from arteries [25]. Therefore, if a DN with low initial signals shows higher signals on T2WI, it is highly suggestive of cancer [1]. An increase in the arterial blood supply can also indicate a risk of DN for carcinogenesis. Dynamic contrast-enhanced MRI can better reflect changes in the arterial blood supply of lesions. Nodules with enhancement in the arterial phase or the appearance of significantly enhanced small nodules in the nodules without obvious enhance- 
ment, i.e., "nodules in nodules", are highly suggestive of the possibility of cancer. Wash-out of contrast agent in the portal venous phase and delayed phase are also highly suggestive of HCC.

Low signals on T1WI and high signals on T2WI are typical manifestations of small HCC. In a small number of cases, small HCCs show high signals on T1WI, which may relate to the composition of fat, copper or glycoprotein. In addition, small HCCs have different pseudocapsules, and the T1WI is sensitive in displaying tumor capsules, showing homogeneous hypo-intense ring of variable thickness. The capsule shows no enhancement at the early stage of dynamic contrast-enhanced MRI. At late stage and during the delayed phase, the capsule may exhibit less regular circular high signal zone, due to the diffusion of contrast agent within the capsule and the long duration of retention and washout of contrast agent in the lesion $[25,26]$.

\subsection{Application of Targeted Contrast Agents for MRI}

In general, hemodynamic changes associated with carcinogenesis of cirrhosis arethe pathological basis of imaging for the detection and diagnosis of HCC. However, HCCs with less abundant or atypical blood supplies are not uncommon. In those cases, it is difficult to make a diagnosis for those lesions using an examination that relies solely on blood flow perfusion changes, such as contrast-enhanced CT and perfusion and contrast-enhanced ultrasound.

The application of nuclear magnetic resonance contrast agent improves the detection rate of HCC. Superparamagnetic iron oxide (SPIO) is a specific type of contrast agent targeting at the reticuloendothelial system. It is taken up mainly by intrahepatic Kupffer cells. Kupffer cells are present in DNs and they can take up SPIO. SPIO is not taken up by HCC nodules, which contain no Kupffer cells. SPIO-containing tissues have decreased T2 values, and they show low signals on T2WI. Tissues containing no SPIO may show high signals on T2WI. MRI can take advantage of this property of SPIO as a contrast agent to reveal the number and function of intrahepatic Kupffer cells, which may help in making an accurate diagnosis of intrahepatic DNs and small HCCs[24]. Gadolinium ethoxybenzyl diethylenetriamine pentaacetic acid (Gd-EOB-DTPA) is a new type of liver cell-specific contrast agent [27], approximately $50 \%$ of which can be taken up by hepatic cells and excreted via the bile duct. The other $50 \%$ is excreted via the kidneys. Therefore, Gd-EOB-DTPA not only is a liver cell-specific contrast agent, but also may cause enhancement of hepatic blood vessels and sinusoids. The contrast agent interacts with proteins after it enters hepatic cells, caus- ing an increase in the micro-viscosity of intracellular environment and increased T1 relaxation time. Gd-EOBDTPA is characterized by high signals on T1WI during the hepatobiliary phase. One report showed that $95.4 \%$ of HCCs have low signals in Gd-EOB-DTPA-enhanced hepatobiliary-phase MRI images [28]. Using Gd-EOBDTPA-enhanced hepatobiliary-phase M-RI images, the detection rates of well-differentiated early HCCs, HCCs with less-abundant blood supplies, and HCCs with diameters $\leq 1 \mathrm{~cm}$ are higher than those using multi-slice spiral CT, CTAP, CTHA, and conventional three-phase dynamic contrast-enhanced MR [29-31].

In a small number of cases, HCCs showed abnormal high signals on T1WI during the hepatobiliary phase. Pathological examination revealed that those cancer cells were well-differentiated and had strong bile secretion function [32], DNs often show an isosignal or high signal during the hepatobiliary phase. Gd-EOB-DTPA, therefore, is of great significance for the differentiation of DNs and early HCCs [27-31]

\section{Conclusions and Prospects}

It is not difficult to make a correct diagnosis of DN and small HCC nodules with typical properties in ultrasound, CT, and MRI images. However, the first-choice method for the early diagnosis of HCC is still an enormous challenge. Currently, imaging results, serum tumor markers, and other clinical data are integrated for the diagnosis in clinical practice. How to find an economical, non-invasive, and accurate method for early diagnosis still requires further study. With the development of molecular imaging, the rules of occurrence and development of diseases and imaging manifestations can be revealed comprehensively and thoroughly at the molecular level. Molecular imaging may help detect the biological characteristics of early-stage tumors, including pre-cancerous molecular changes, genetic changes, tumor cell markers, and growth dynamics. This may provide a new method for the early diagnosis of HCC.

Recently, there are some methods used to detect the malignant tumor tissues from normal surrounding tissue. One recent patent provided an apparatus to detect the change in xenon concentration in the tissue of the liver by using an X-ray CT system [33]. The hepatic arterial blood flow amount and the portal blood flow amount are determined by the xenon concentration, furthermore, to estimate the blood flow of malignant tumor tissues. Second patent provided the new contrast media encapsulated within $50 \mathrm{~nm}$ to $10 \mu \mathrm{m}$ microspheres to improve contrast ratio in the liver to tumor in CT images [34]. This contrast media also resulted in sustained contrast enhancement $1 \mathrm{~h}$ after administration, providing the opportunity to use a lesser X-ray exposure in a single scan to discrimi- 
nate cancerous tissue from normal surrounding tissue. Third patent described a method for detecting a halo surrounding an ultrasound image of a tumor by detecting the boundary of the tumor and defining two annular regions around that boundary. In this method, presence of this halo is indicative of a malignant tumor [35].

\section{Acknowledgements}

The authors were partially supported by Chinese State Administration of Foreign Experts Affairs (CG2010230004) and Kentucky Lung Cancer Research Program Award (cycle 9). The authors have no conflict of interest relevant to this article. This work was supported by grants from the Natural Science Foundation of Heilongjiang Province (No. D2009-05) and the Educational Committee of Heilongjiang Province (No.11541166).

\section{REFERENCES}

[1] N. D. Theise, "Macroregenerative (Dysplastic) Nodules and Hepatocarcinogenesis: Theoretical and Clinical Considerations," Seminars in Liver Disease, Vol. 15, No. 4, 1995, pp. 360-371. doi:10.1055/s-2007-1007287

[2] "Pathologic Diagnosis of Early Hepatocellular Carcinoma: A Report of the International Consensus Group for Hepatocellular Neoplasia," Hepatology, Vol. 49, No. 2, 2009, pp. 658-664. doi:10.1002/hep.22709

[3] G. L. Bennett, G. A. Krinsky, R. J. Abitbol, S. Y. Kim, N. D. Theise and L. W. Teperman, "Sonographic Detection of Hepatocellular Carcinoma and Dysplastic Nodules in Cirrhosis: Correlation of Pretransplantation Sonography and Liver Explant Pathology in 200 Patients," American Journal of Roentgenology, Vol. 179, No.1, 2002, pp. 7580 .

[4] S. Tanaka, T. Kitamura, M. Fujita, K. Nakanishi and S. Okuda, "Color Doppler Flow Imaging of Liver Tumors," American Journal of Roentgenology, Vol. 154, No. 3, 1990, pp. 509-514.

[5] T. Hosoki, M. Mitomo, S. Chor, N. Miyahara, M. Ohtani and K. Morimoto, "Visualization of Tumor Vessels in Hepatocellular Carcinoma. Power Doppler Compared with Color Doppler and Angiography," Acta Radiologica, Vol. 38, No. 3, 1997, pp. 422-427. doi:10.1080/02841859709172094

[6] K. Koito, T. Namieno and K. Morita, "Differential Diagnosis of Small Hepatocellular Carcinoma and Adenomatous Hyperplasia with Power Doppler Sonography," American Journal of Roentgenology, Vol. 170, No. 1, 1998, pp. 157-161.

[7] V. Catala, C. Nicolau, R. Vilana, M. Pages, L. Bianchi, M. Sanchez and C. Bru, "Characterization of Focal Liver Lesions: Comparative Study of Contrast-Enhanced Ultrasound versus Spiral Computed Tomography," European Radiology, Vol. 17, No. 4, 2007, pp. 1066-1073. doi:10.1007/s00330-006-0444-6

[8] R. Li, Y. Guo, X. Hua, Y. He, J. Ding, A. Guo and M.
Luo, "Characterization of Focal Liver Lesions: Comparison of Pulse-Inversion Harmonic Contrast-Enhanced Sonography with Contrast-Enhanced CT," Journal of Clinical Ultrasound, Vol. 35, No. 3, 2007, pp. 109-117. doi:10.1002/jcu.20310

[9] L.P. Larsen, M. Rosenkilde, H. Christensen, N. Bang, L. Bolvig, T. Christiansen and S. Laurberg, "The Value of Contrast Enhanced Ultrasonography in Detection of Liver Metastases from Colorectal Cancer: A Prospective Double-Blinded Study," European Journal of Radiology, Vol. 62, No. 2, 2007, pp. 302-307. doi:10.1016/j.ejrad.2006.11.033

[10] H. J. Jang, T. K. Kim and S. R. Wilson, "Small Nodules $(1-2 \mathrm{~cm})$ in Liver Cirrhosis: Characterization with Contrast-Enhanced Ultrasound," European Journal of Radiology, Vol. 72, No. 3, 2009, pp. 418-424. doi:10.1016/j.ejrad.2008.08.011

[11] M. H. Chen, Y. Dai, K. Yan, Z. H. Fan, S. S. Yin, W. Yang, W. Wu, Y. B. Wang and J. Y. Li, "The Role of Contrast-Enhanced Ultrasound on the Diagnosis of Small Hepatocellular Carcinoma $(</=3 \mathrm{~cm})$ in Patients with Cirrhosis," Hepatology Research: The Official Journal of the Japan Society of Hepatology, Vol. 35, No. 4, 2006, pp. 281-288. doi:10.1016/j.hepres.2006.04.013

[12] J. F. Xu, H. Y. Liu, Y. Shi, Z. H. Wei and Y. Wu, "Evaluation of Hepatocellular Carcinoma by Contrast-Enhanced Sonography: Correlation with Pathologic Differentiation," Journal of Ultrasound In Medicine: Official Journal of the American Institute of Ultrasound in Medicine, Vol. 30, No. 5, 2011, pp. 625-633.

[13] G. J. Liu, H. X. Xu, M. D. Lu, X. Y. Xie, Z. F. Xu, Y. L. Zheng and J. Y. Liang, "Correlation between Enhancement Pattern of Hepatocellular Carcinoma on Real-Time Contrast-Enhanced Ultrasound and Tumour Cellular Differentiation on Histopathology," The British Journal of Radiology, Vol. 80, No. 953, 2007, pp. 321-330. doi: $10.1259 / \mathrm{bjr} / 54805002$

[14] P. Hytiroglou, Y. N. Park, G. Krinsky and N. D. Theise, "Hepatic Precancerous Lesions and Small Hepatocellular Carcinoma," Gastroenterology Clinics of North America, Vol. 36, No. 4, 2007, pp. 867-887. doi:10.1016/j.gtc.2007.08.010

[15] M. Kudo, "Hepatocellular Carcinoma 2009 and beyond: From the Surveillance to Molecular Targeted Therapy," Oncology, Vol. 75, Suppl. 1, 2008, pp. 1-12. doi:10.1159/000181865

[16] M. Kudo, K. Hatanaka and K. Maekawa, "Newly Developed Novel Ultrasound Technique, Defect Reperfusion Ultrasound Imaging, Using Sonazoid in the Management of Hepatocellular Carcinoma," Oncology, Vol. 78, Suppl. 1, 2010, pp. 40-45. doi:10.1159/000315229

[17] V. Salvatore, A. Borghi and F. Piscaglia, "Contrast-Enhanced Ultrasound for Liver Imaging: Recent Advances," Current Pharmaceutical Design, Vol. 18, No. 15, 2012, pp. 2236-2252. doi:10.2174/138161212800099892

[18] J. H. Lim, C. K. Kim, W. J. Lee, C. K. Park, K. C. Koh, S. W. Paik and J. W. Joh, "Detection of Hepatocellular Carcinomas and Dysplastic Nodules in Cirrhotic Livers: Accuracy of Helical CT in Transplant Patients," American 
Journal of Roentgenology, Vol. 175, No. 3, 2000, pp. 693698.

[19] K. Takayasu, Y. Muramatsu, Y. Mizuguchi and H. Ojima, "CT Imaging of Early Hepatocellular Carcinoma and the Natural Outcome of Hypoattenuating Nodular Lesions in Chronic Liver Disease," Oncology, Vol. 72, Suppl. 1, 2007, pp. 83-91. doi:10.1159/000111712

[20] M. Hayashi, O. Matsui, K. Ueda, Y. Kawamori, M. Kadoya, J. Yoshikawa, T. Gabata, T. Takashima, A. Nonomura and Y. Nakanuma, "Correlation between the Blood Supply and Grade of Malignancy of Hepatocellular Nodules Associated with Liver Cirrhosis: Evaluation by CT during Intraarterial Injection of Contrast Medium," American Journal of Roentgenology, Vol. 172, No. 4, 1999, pp. 969-976.

[21] P. V. Pandharipande, G. A. Krinsky, H. Rusinek and V. S. Lee, "Perfusion Imaging of the Liver: Current Challenges and Future Goals," Radiology, Vol. 234, No. 3, 2005, pp. 661-673. doi:10.1148/radiol.2343031362

[22] Y. Tsushima, S. Funabasama, J. Aoki, S. Sanada and K. Endo, "Quantitative Perfusion Map of Malignant Liver Tumors, Created from Dynamic Computed Tomography Data," Academic Radiology, Vol. 11, No. 2, 2004, pp. 215223. doi:10.1016/S1076-6332(03)00578-6

[23] D.V. Sahani, N.S. Holalkere, P.R. Mueller and A.X. Zhu, "Advanced Hepatocellular Carcinoma: CT Perfusion of Liver and Tumor Tissue-Initial Experience," Radiology, Vol. 243, No. 3, 2007, pp. 736-743. doi:10.1148/radiol.2433052020

[24] L. Macarini, P. Milillo, A. Cascavilla, G. Scalzo, L. Stoppino, R. Vinci, G. Moretti and G. Ettorre, "MR Characterisation of Dysplastic Nodules and Hepatocarcinoma in the Cirrhotic Liver with Hepatospecific Superparamagnetic Contrast Agents: Pathological Correlation in Explanted Livers," La Radiologia Medica, Vol. 114, No. 8, 2009, pp. 1267-1282. doi:10.1007/s11547-009-0464-9

[25] Y.Y. Jeong, N. Y. Yim and H. K. Kang, "Hepatocellular Carcinoma in the Cirrhotic Liver with Helical CT and MRI: Imaging Spectrum and Pitfalls of Cirrhosis-Related Nodules," American Journal of Roentgenology, Vol. 185, No. 4, 2005, pp. 1024-1032. doi:10.2214/AJR.04.1096

[26] C. D. Witjes, F. E. Willemssen, J. Verheij, S. J. van der Veer, B. E. Hansen, C. Verhoef, R. A. de Man and J. N. Ijzermans, "Histological Differentiation Grade and Microvascular Invasion of Hepatocellular Carcinoma Predicted by Dynamic Contrast-Enhanced MRI," Journal of Magnetic Resonance Imaging, Vol. 36, No. 3, 2012, pp.
641-647. doi:10.1002/jmri.23681

[27] A. Frydrychowicz, M. G. Lubner, J. J. Brown, E. M. Merkle, S. K. Nagle, N. M. Rofsky and S. B. Reeder, "Hepatobiliary MR Imaging with Gadolinium-Based Contrast Agents," Journal of magnetic Resonance Imaging, Vol. 35, No. 3, 2012, pp. 492-511. doi:10.1002/jmri.22833

[28] H. Y. Sun, J. M. Lee, C. I. Shin, D. H. Lee, S. K. Moon, K. W. Kim, J. K. Han and B. I. Choi, "Gadoxetic AcidEnhanced Magnetic Resonance Imaging for Differentiating Small Hepatocellular Carcinomas $(<$ or $=2 \mathrm{~cm}$ in Diameter) from Arterial Enhancing Pseudolesions: Special Emphasis on Hepatobiliary Phase Imaging," Investigative Radiology, Vol. 45, No. 2, 2010, pp. 96-103. doi:10.1097/RLI.0b013e3181c5faf7

[29] S. S. Ahn, M. J. Kim, J. S. Lim, H. S. Hong, Y. E. Chung and J. Y. Choi, "Added Value of Gadoxetic Acid-Enhanced Hepatobiliary Phase MR Imaging in the Diagnosis of Hepatocellular Carcinoma," Radiology, Vol. 255, No. 2, 2010, pp. 459-466. doi:10.1148/radiol.10091388

[30] M. J. Kim, "Current Limitations and Potential Breakroughs for the Early Diagnosis of Hepatocellular Carcima," Gut and Liver, Vol. 5, No. 1, 2011, pp. 15-21. doi:10.5009/gnl.2011.5.1.15

[31] M. Kudo, "The 2008 Okuda Lecture: Management of Hepatocellular Carcinoma: From Surveillance to Mocular Targeted Therapy," Journal of Gastroenterology and Hepatology, Vol. 25, No. 3, 2010, pp. 439-452. doi:10.1111/j.1440-1746.2009.06207.x

[32] S. A. Lee, C. H. Lee, W. Y. Jung, J. Lee, J. W. Choi, K. A. Kim and C. M. Park, "Paradoxical High Signal Intensity of Hepatocellular Carcinoma in the Hepatobiliary Phase of Gd-EOB-DTPA Enhanced MRI: Initial Experience,” Magnetic Resonance Imaging, Vol. 29, No. 1, 2011, pp. 83-90. doi:10.1016/j.mri.2010.07.019

[33] S. Shigeru, "Method and Apparatus for Measuring Hepatic Blood Flow Amount," US Patent No. 6176838, 2001.

[34] See and R. Jackie, "Methods of Preparing Micro Encapsulated Agents for Use in the Detection of Tumors by CT Imaging," US Patent No. 6001333, 1999.

[35] X. H. Hao, J. W. Charboneau, N, J, Hangiandreou and J. F. Greenleaf, "Detection of Tumor Halos in Ultrasound Images," US Patent No. 6984211, 2006. 